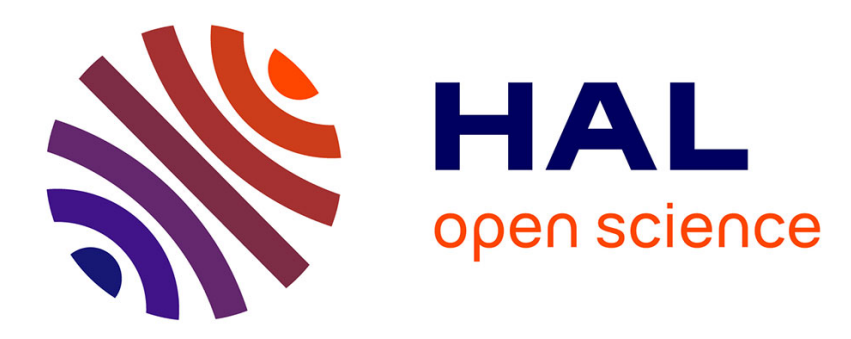

\title{
Connectivity properties in complex systems
}

A. Coniglio

\section{To cite this version:}

A. Coniglio. Connectivity properties in complex systems. Journal de Physique IV Proceedings, 1993, 03 (C1), pp.C1-1-C1-13. 10.1051/jp4:1993101 . jpa-00251544

\section{HAL Id: jpa-00251544 https://hal.science/jpa-00251544}

Submitted on 1 Jan 1993

HAL is a multi-disciplinary open access archive for the deposit and dissemination of scientific research documents, whether they are published or not. The documents may come from teaching and research institutions in France or abroad, or from public or private research centers.
L'archive ouverte pluridisciplinaire HAL, est destinée au dépôt et à la diffusion de documents scientifiques de niveau recherche, publiés ou non, émanant des établissements d'enseignement et de recherche français ou étrangers, des laboratoires publics ou privés. 


\title{
Connectivity properties in complex systems
}

\author{
A. CONIGLIO \\ Dipartimento di Scienze Fisiche, Universitd di Napoli, Mostra d'Oltremare Pad. 19, 80125 Napoli, Italy
}

After a brief review of random and correlated percolation a new model called frustrated percolation is introduced. This model which contains, as essential ingredients connectivity and frustration, applies to complex systems as spin glasses and gels. The properies of the model are studied using renormalization group approach. Two critical points are found, one at a temperature $T_{p}$ caracterized by a percolation transition with critical exponents of the ferromagnetic $1 / 2$ state Potts model, the other at a lower temperature $T_{s g}$ correspondes to a spin glass transition. These results are compared with the glasslike behaviour found by Adam et al in a polymeric cluster solution near the gelation threshold.

Après un bref resumé sur la percolation aléatoire et correlée nous introduisons un nouveau modèle appellé percolation frustrée. Ce modéle qui contient comme ingrédients essentiels la connexité e la frustration s'applique aux systémes complexes comme le verres de spin e de gels. Les propriétés du modèle sont étudiées par l'approche du groupe de rénormalisation. Nous trouvons deux points critiques, un premier a la température $T_{p}$ caractérisé par une transition de percolation avec les exposants du modéle ferromagnetique de Potts à $1 / 2$ état, l'autre à une température plus basse $T_{s g}$ qui correspond à une transition de verres de spins. Ces resultats sont comparés avec le comportament du type verre trouvé par Adam et al dans une solution d'amas de polymères proche du seuil de gélification.

The dicovery of the basic laws governing the behaviour of complex systems is one of the big challenge in physics. From here the necessity to adapt old concepts and models, which have been useful to study simple systems, in order to be applied to more complex phenomena. 
Percolation theory ${ }^{1}$ has played a central role in many problems, in which connectivity is the basic feature of the physical phenomena involved. This is the case for random resistor network, dilute feromagnets at zero temperature, flow of a fluid in a porous media. Even in temperature dependent phenomena like phase transitions the phenomenon can be mapped onto a percolation problem once the proper cluster is identified. ${ }^{2,3}$

In more complex systems, such as spin glasses ${ }^{4}$, an important role is played by frustration. To describe such phenomena in terms of percolation concepts, we have introduced a new model ${ }^{5}$, which we call frustrated percolation. This model contains, as main ingredients, both connectivity and frustration. After a brief introduction to random and correlated percolation, the properties of the frustrated percolation will be discussed along with its applications to spin glasses and gels.

\section{Random and correlated percolation.}

In random bond percolation one assignes a probability $p$ to each bond of a d-dimensionasl hypercubic lattice of beeing present. The bonds are statistically indipendent therefore the weight of a given configuration of bonds $C$ is given by

$$
W(C)=p^{|C|}(1-p)^{|A|}
$$

where $|C|$ is the number of present bonds and $|A|$ the number of absent bonds. Random percolation applies when connectivity plays the major role and correlations in the system can be neglected.

In many problems both correlations and connectivity play an important role. Consider for example an Ising model with nearest neighbour interaction $J$ with the hamiltonian given by

$$
-H=J \sum_{\langle i j\rangle}\left(S_{i} S_{j}-1\right),
$$

where $S_{i}= \pm 1$ and the sum is over the nearest neighbour pairs of spins. If we want to characterize the Ising transition in terms of geometrical clusters we have to define the 
clusters in a suitable way in such a way that the percolation transition coincides with the Ising critical point. It is well known that in an Ising model the naive definition of clusters made of nearest neighbor parallel spins is not satisfactory. These clusters are in fact too large, representing both correlations and pure geometrical effects. As an example at $T=\infty$ in a typical configuration clusters of parallel spins are present although there is total absence of correlations. To eliminate the pure geometrical effect a different definition of cluster was proposed ${ }^{3}$ for the Ising model. The idea was based on a site bond correlated percolation model which was introduced as a model for a sol-gel transition. ${ }^{6}$

Consider an Ising configuration of spins: between $\mathrm{nn}$ parallel spins, introduce a bond being active with probability $p$. The bonds do not affect the energy of the pair of the spins, instead they are introduced to define the connectivity and therefore the clusters. These are defined as maximal sets of nn parallel spins connected by bonds (Fig. 1).

(a)

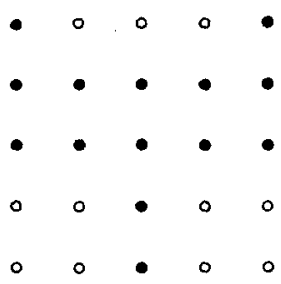

(b)

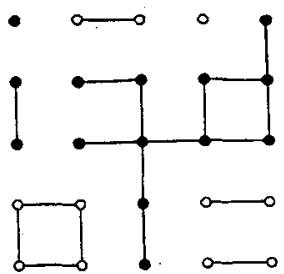

Fig. 1: (a) Ising configuration: "up" spins and "down" spins are represented respectively by filled and empty circles. (b) Correct clusters obtained from the configuration given in (a) by putting bonds between sites in the same state with probability $p=1-e^{-2 \beta J}$.

How can we calculate the connectivity and thermal properties of the Ising model? The weight for a given configuration of spin $\left\{S_{i}\right\}$ is given by the Boltzmann factor $e^{-\beta H\left\{S_{i}\right\}}$, where $\beta=1 / k T$. The weight associated with a given configuration of bonds $C$ which is made of $|C|$ bonds present and $|B|$ bonds absent between parallel spins, is given by $p^{|C|}(1-p)^{|B|}$. Therefore the weight associated to a given configuration of spins $\left\{S_{i}\right\}$ and 
bonds $C$ is

$$
W\left(C,\left\{S_{i}\right\}\right)=p^{|C|}(1-p)^{|B|} e^{-\beta H\left\{S_{i}\right\}}
$$

Any connectivity quantity can be calculated using (2). For example the pair connectedness $p_{i j}$ defined as the probability that $i$ and $j$ are in the same finite cluster is $p_{i j}=\left\langle\gamma_{i j}\right\rangle$, where $\gamma_{i j}=1$ or 0 depending whether or not $i$ and $j$ are in the same finite cluster and the brackets stand for the average over all configurations of spins and bonds weighted with (2).

Note than when the average is applied to a thermal quantity $f\left\{S_{i}\right\}$ it reduces to the usual average weighted only with the Boltzmann factor $\exp \left\{-\beta H\left\{S_{i}\right\}\right\}$ since $f\left\{S_{i}\right\}$ does not depend on the bond configuration and $\sum_{C} p^{|C|}(1-p)^{|B|}=1$. To study the connectivity properties of this model it was originally ${ }^{3}$ considered an Hamiltonian formalism based on a dilute Potts model. This Hamiltonian formalism allowed to study the problem using the Migdal-Kadanoff renormalization group ${ }^{3}$. Moreover from the Hamiltonian formalism it was possible to study the problem for any value of the bond probability $p$ and it was possible to show that for the particular choice of the bond probability

$$
p=1-e^{-2 \beta J}
$$

the size of the clusters diverge at the Ising critical point with Ising critical exponents.

Using the value given by (4) it is possible to express the weight (3) in a more suggestive form. In fact by noting that $e^{-\beta H\left\{S_{i}\right\}}=\left(e^{-2 \beta J}\right)^{|D|}$, where $|D|$ is the number of antiparallel spins, by summing up over all the spin variable compatible with the bond configuration $C$ $W(C)=\sum_{\left\{S_{i}\right\}} W\left(C,\left\{S_{i}\right\}\right)$ it is easy to prove that ${ }^{5}$

$$
W(C)=p^{|C|}(1-p)^{|A|} q^{N(C)},
$$

where $q=2$ and $N(C)$ is the number of clusters in the bond configuration $C$. Note that the weight (5) differs from the weight of random percolation (1) by an extra factor of $2^{N(C)}$. The percolation problem with a weight (5) and $q=2$ has a percolation transition with exponents of the Ising model. For general $q$ the percolation transition has exponents 
of the ferromagnetic $q$-state Potts model. And the sum over all the bond configuration in (5) gives the partition function of the $q$-state Potts model.

$$
Z=\sum_{C} p^{|C|}(1-p)^{|B|} q^{N(C)}
$$

This is the in fact the cluster formulation of the partition function of the $q$-state Potts model developed by Fortuin and Kasteleyn. ${ }^{7}$ Note that in this formulation taking the limit $q=1$ in (5) one recoveers the weight of the random percolation problem.

Recently Swendsen and Wang ${ }^{14}$ have used these clusters to build a dynamics which drastically reduces the critical slowing down near the critical point.

\section{Application to gels.}

Strong Gels or irreversible gels are characterized by the fact that when two monomers react they form a chemical permanent bond. The transition from the sol phase to the Gel phase is well described by random percolation. More complicated is the case when the monomers interact forming not permanent bonds. In this case a simple model ${ }^{6}$ based on correlated percolation, has been introduced to describe the interplay between phase separation and the formation of a gel. This model is described by a binary mixture or lattice gas in which two nearest neighbour monomers interact with two type of energies one corresponding to a Van der Wals type of interaction and a second stronger energy associated to a chemical interaction.

On very long time scale the momnomers feel one effective interacting energy, and the system behave as a normal fluid. On short time scale the strong energy bonds can be considered as permanent and the formation of large clusters leads to an extremely high viscosity responsable for the gel transition.

The phase diagram in the long time regime, after equilbrium has been reached, has been calculated in mean field in the more general case in which the system is made of long polymer chains in a solvent. The result is shown in Fig. (2) for different values of 
the parameters. The coexistence curve has been drawn toghether with the gelation curve which corresponds to the formation of an infinite cluster made of stong bonds. Along the line the critical exponents correspond to random percolation $(q=1)$ except when (Fig. $2 b)$ the percolation line ends at the consolute point. At this particular point the critical behaviour is given by the Ising critical exponents. $(q=2)$. The data are consistent with the experimental result of T.Tanaka et $\mathrm{al}^{8}$ on gelatin/water/methanol mixture.



(a)

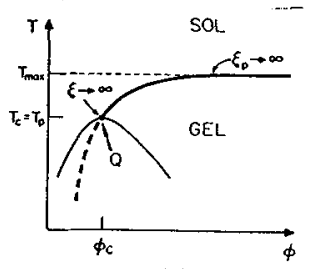

(b)

Fig. 2: (a) Coexistence curve for the polymer solvent binary mixture and sol-gel phase boundary for two different solvent. The broken curve is the continuation of the geletaion curve into the two-phase region. The solvent have been chosen in such a way that the consolute point is (a) in the sol phase, (b) on the gelation curve. (After Ref.[6]).

More recently the kinetics of this model has been studied, via Monte Carlo computer simulation, in a quench from high temperature to low temperatrure below the consolute point. The study of the factor structure shows a behaviour which for short time follows the kinetics of the standard lattice gas model. While after a crossover time $t_{\times}$the system is stuck and no more evolution occurs. The data compare favorably with results of experiments on simultaneous gelation and phase separation in gelatin/water/methanol mixtures. ${ }^{9}$

In a more complex case random or correlated percolation may not be suficient to model gelation. This is the case when frustration is present. Consider for simplicity the case in wich monomers have valence three. In this case there are situations when not all the interactions can be satisfied. To study such case a new percolation model ${ }^{5}$ has been 
introduced, which contains frustration as essential ingredient. To introduce such model we proceed along the following steps:

1) First construct a cluster formalism for the partition function of the spin glass model.

2) Generalize the partition function to spin with q-states.

3) take the limit $q \rightarrow 1$.

Note that this procedure in the case of no frustration corresponds to write the free energy of the q-state Potts model using the cluster formalism of Kasteleyn and Fortuin as in Eq. (6) and take then the limit $q \rightarrow 1$.

\section{Clusters in Spin Glasses}

Consider the nn Ising Spin Glass model:

$$
-H=\sum_{\langle i j\rangle}\left(J_{i j} S_{i} S_{j}-J\right)
$$

where the nn interactions $J_{i j}= \pm J$ are randomly distributed. For a given set of interactions $\left\{J_{i j}\right\}$ the partition function is given by

$$
Z\left\{J_{i j}\right\}=\sum_{\left\{S_{i}\right\}} e^{-\beta H} .
$$

A concept crucial in spin glasses is frustration. This occurs when the positive and negative interactions are distributed in such a way that not all the spins can satisfy all the interactions in such a way that $J_{i j} S_{i} S_{j}>0$ (Fig. 3a).

The average magnetization at site $i$ is given by $m_{i}=\left\langle S_{i}\right\rangle$ where $\langle\ldots\rangle$ stand for the thermal average for a fixed configuration of interactions $J_{i j}$. For high temperature $m_{i}=0$. For low temperature the spin glass phase is characterized by the Edwards-Anderson order parameter:

$$
q_{E A}=1 / N \sum_{i} \overline{\left\langle S_{i}\right\rangle^{2}}
$$

where $N$ is the number of spins and the bar stands for the average over all the interaction configuration. $q_{E A}$ is different from zero in the spin glass phase and goes to zero at the spin glass temperature $T_{s g}$. 


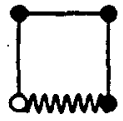

(a)

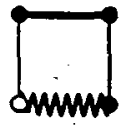

(b)

Fig. 3: Example of a frustrated plaquette. "Up" spins and "down"spins are represented rispectively by filled and empty circles. Straight and wavy lines indicate positive and negative interactions, respectively. (b) Clusters obtained from configuration given in (a) by putting bonds (heavy lines) between spins satisfying the interaction with probability $p=1-e^{-2 \beta J}$.

Spin glasses have another peculiarity already above $T_{s g}$. It is found in fact that the autocorrelation function of a typical quantity $A$ decays with a stretched exponential between $T_{s g}$ and $T^{*}$, a temperature close to the critical temperature of the ferromagnetic Ising model, i.e.

$$
f(t)=<A\left(t+t^{\prime}\right) A(t)>-<A(t)>^{2}
$$

behaves as

$$
f(t) \sim \exp -(t / \tau)^{\beta(T)}
$$

with $\beta(T)$ function of $T$ which assumes a value from 0.3 to 1 for temperature between $T_{s g}$ and $T^{*}$

Finally strong hysteresis effect, with respect to a magnetic field, characterize further the spin glass phase below $T_{s g}$.

Now I want to illustrate how the cluster definition given for the ferromagnetic Ising model can be extended ${ }^{5}$ to the Ising Spin Glass model. Since SG order propagates through "satisfied" interactions it is natural to define clusters by randomly putting bonds only between those spins that satisfy the interaction, i.e., we put bonds between only those spins for which $J_{i j} S_{i} S_{j}>0$ (see Fig. $3 \mathrm{~b}$ ). The bonds are put randomly with probability 
$p=1-e^{-2 \beta J}$. Two spins are in the same cluster if they are connected by at least one chain of bonds.

It is possible to prove ${ }^{5}$ that for a given configuration of interactions:

$$
\left\langle S_{i}\right\rangle=P_{i \uparrow}-P_{i \downarrow}
$$

$P_{i \uparrow}\left(P_{i \downarrow}\right)$ is the probability that $i$ is up (down) and belongs to the infinite cluster.

From (10) we can construct the Edwards-Anderson order parameter and relate it to the connectivity properties

$$
q_{E A} \equiv \frac{1}{N} \sum_{i} \overline{\left\langle S_{i}>^{2}\right.}=\frac{1}{N} \sum_{i} \overline{\left(P_{i \uparrow}-P_{i \downarrow}\right)^{2}}
$$

where $N$ is the number of sites and the bar stands for the average over the realization of interactions. Moreover, we can also evaluate the density of sites in the infinite cluster $\rho^{\text {inf }}$

$$
\rho^{\mathrm{inf}}=\frac{1}{N} \sum_{i} \overline{\left(P_{i \uparrow}-P_{i \downarrow}\right)}
$$

which goes to zero at a percolation temperature $T_{p}$. Since

$$
q_{E A} \leq \rho^{\mathrm{inf}}
$$

it follows $T_{s g} \leq T_{p}$

Numerically in $3 d$ it is found ${ }^{11} T_{s g} \simeq 1.2$, whereas the percolation transition is found to be much larger $T_{p} \simeq 3.95,{ }^{12}$ very close to the temperature $T^{*}$.

Finally the partition function for each configuration of interaction $\left\{J_{i j}\right\}$ can be written in the formalism of Fortuin and Kasteleyn as

$$
Z\left\{J_{i j}\right\}=\sum_{C} W(C)
$$

where

$$
W(C)=p^{|C|}(1-p)^{|B|} q^{N(C)}
$$


if the configuration of bonds $C$ does not contain any frustrated loop, and

$$
W(C)=0 \quad \text { otherwise }
$$

In (15) $q=2$. The partition function (14) of the Ising spin glass model, in this cluster formulation, is very similar to the partition function of the ferromagnetic Ising model, except that in the spin glass case due to (15) the sum in (14) runs only on those bond configurations which do not contain frustration.

\section{Frustrated percolation.}

In the previous Sec. we have introduced the cluster formulation of the partition function of an Ising spin glass, which is given by (14) with $q=2$. This partion function can be immediately generalized to any $q$ and it can be shown ${ }^{13}$ to be obtained by the following Hamiltonian:

$$
-H=\sum_{<i j>}\left[J \delta_{\sigma_{i} \sigma_{j}}\left(\epsilon_{i j} S_{i} S_{j}+1\right)-2 J\right]
$$

where at each site there is a Ising variable $S_{i}= \pm 1$ and an $s$-state Potts variable $\sigma_{i}=$ $1, \ldots, s$. The $\epsilon_{i j}= \pm 1$ are quenched variables and specify the sign of the interaction $J_{i j}=\epsilon_{i j} J$ of the Ising variables. The partition function of (16) can be written in the form (14) with $q=2 s$. Finally if we take the limit $q \rightarrow 1$ we obtain the frustrated percolation model.

For clarity we define the frustrated percolation model in a more direct way:

1) Assigne at random a configuration of interaction $\left\{J_{i j}\right\}$ on a lattice.

2) Assigne to any configuration $C$ of bonds a weight given by (15) with $q=1$, i.e.

$$
W(C)=p^{|C|}(1-p)^{|B|}
$$

if the configuration of bonds $C$ does not contain any frustrated loop, and

$$
W(C)=0 \quad \text { otherwise. }
$$


A loop is frustrated if contains an odd number of negative $J_{i j}$. Note that the bond probability is related to the strength of interaction $J$ via Eq.(5). However one could also fix $J=1$ and use $p$ as an indipendent variable.

For each configuration of interactions $\left\{J_{i j}\right\}$ one has to calculate the percolation quantities with the weight given by (17) and then average over all interaction configurations. If there is no frustration then the model gives back the weight of standard percolation (1).

To illustrate the difference between standard and frustrated percolation, consider a frustrated plaquette. All bond configurations are allowed having the same weight as in standard percolation, except the one with all four bonds present, which has zero weight in frustrated percolation.

To find the properties of the frustarted percolation model we have solved ${ }^{14}$ the hamiltonian (5) in the limit $q \rightarrow 1$ model on a hyerarchical lattice, introduced by Mckay et $\mathrm{al}^{15}$ to study the spin glass transition.

The results are quite interesting. We find the existence of two critical points. One at a percolation temperature $T_{p}$ and another at a lower temperature $T_{s g}$. The critical behaviour at $T_{p}$ is in the same universality class of a ferromagnetic $1 / 2$-state Potts. (as opposed to standard percolation which is decribed by the ferromagnetic 1-state Potts model). The transition at $T_{s g}$ is in the same universality class as the Ising spin glass transition.

Summarizing, we should expect 2 critical points $T_{p}$ and $T_{s g}$ in systems for which frustrated percolation is relevant.

1) A percolation transition at $T_{p}$ with critical exponents of the $1 / 2$-state Potts model, with exponential relaxation above $T_{p}$.

2) A spin glasss transition at $T_{s g}$ with power law relaxation behaviour below $T_{s g}$, and all the fenomenology related to the spin glass transition, in particular hysteresis effects which should be amenable to direct measurements.

3) A stretched exponential regime between $T_{s g}$ and $T^{*}$ close to $T_{p}$.

Adam et al $^{16}$ have recently reported a dynamical study of a polymer cluster solution 
near the gelation threshold. In this experiment they first obtained a cluster distribution by chemical gelation, then by dilution they changed the polymer concentration $c$. They found two "critical concentrations": $c^{*}$ ( the concentration when the polymer chains start to interpenetrate) and a second freezing concentration $c_{f}$.

1) Above $c^{*}$ they found exponential relaxation behaviour.

2) Below $c_{f}$ a regime with a power law relaxation behaviour.

3) Between $c_{f}$ and $c^{*}$ a stretched exponential behaviour.

The presence of the two critical concentration and the strong similarity with the spin glass phenomenology, is strongly suggestive of a description in terms of the frustrated percolation model.

In conclusion after a review on random and correlated percolation we have described a new frustrated percolation model which may be relevant to a large class of phenomena driven by the interplay between connectivity and frustration.

\section{ACKNOWLEDGMENTS}

I wish to thank V. Cataudella, L. de Arcangelis, F. di Liberto, S.C.Glotzer, M.F.Gyure, G. Monroy, F. Peruggi, U. Pezzella, S. Prakash, F. Sciortino and H.E. Stanley, for collaboration in this field. This work was supported in part by grants MURST and CNR.

[1] D. Stauffer and A. Aharony, Introduction to percolation theory second edition (London: Taylor and Francis) 1985.

[2] C. M. Fortuin and P. W. Kasteleyn, Physica (Utrecht) 57, 536 (1972).

[3] A. Coniglio and W. Klein, J. Phys. A 12, 2775 (1980).

[4] Binder K. and Young A.P., Rev. Mod. Phys. 58, 801 (1986).

[5] Coniglio A., di Liberto F., Monroy G. and Peruggi F., Phys. Rev.B 44, 2605 (1991).

[6] A. Coniglio, H. E. Stanley and W. Klein, Phys. Rev. Lett. 42, 518 (1979).

[7] R. H. Swendsen and J. S. Wang, Pliys. Rev. Lett 58, 86 (1987), Wang J.S. and Swendsen R.H., Physica A 6,249 (1990). 
[8] T. Tanaka, G. Swislow and I. Ohmine, Phys. Rev. Lett. 42, 1557 (1979).

[9] S.C. Glotzer, M. Gyure, F. Sciortino, A.Coniglio and H.E. Stanley to be published.

[10] R. Bansil, J. Lal and B.L. Carvalho, Polymer 33, 2961, (1992).

[11] Ogielski A.T., Phys. Rev. B 37, 7384 (1985).

[12] de Arcangelis L., Coniglio A. and Peruggi F., Europhys. Lett. 14, 515 (1991).

[13] V. Cataudella, A. Coniglio, L. de Arcangelis and F. di Liberto, Physica A in press.

[14] U. Pezzella and A. Coniglio to be published.

[15] S.R. Mckay, A.N. Berker and S. Kirkpatrick, Phys. Rev. Lett. 48, 767 (1982).

[16] M. Adam, M, Delsanti J.P.Munch and D. Durand, Phys. Rev. Lett. 61, 706, 1988. 